\title{
Estado Actual de las Competencias Científico Naturales desde el Aprendizaje por Indagación
}

\author{
Diana Milena Fuentes Galvis* \\ Andrea Puentes López** \\ Gustavo Adolfo Flórez Restrepo***
}

Artículo de investigación

Fecha de Recepción: 12 julio 2018.

Fecha de Aprobación: 29 octubre 2018.

\section{Resumen}

Este artículo presenta resultados preliminares de una investigación desarrollada en la Institución Educativa Jesús Emilio Jaramillo Monsalve de Chita Boyacá, la cual busca fortalecer las competencias científico naturales de los estudiantes de primaria a través del desarrollo de estrategias basadas en el Aprendizaje por Indagación.

La metodología se fundamentó en la investigación cualitativa, al tratarse de un proceso que comprende la realidad del aula, como fruto de una construcción social. La investigación acción fundamentada en la autorreflexión, sobre las prácticas y sobre las diferentes situaciones que constituyen el proceso en un contexto escolar rural.

Esteprimermomentodereconocimiento, incluyó la caracterización de elementos que se incorporan al proceso de enseñanza-aprendizaje alrededor de las competencias científico naturales. Para dicho proceso se establecieron tres categorías, Escuela Nueva, competencias Científico Naturales y Aprendizaje por Indagación, sobre las cuales se construyeron dos instrumentos de indagación, y el análisis de resultados a partir de la triangulación entre estamentos y marco teórico conceptual.
* Institución Educativa Jesús Emilio Jaramillo Monsalve Boyacá - Colombia temis297@gmail.com ** Institución Educativa Jesús Emilio Jaramillo Monsalve - Boyacá -

Colombia

hcandrea@hotmail.com

*** Universidad Pedagógica y Tecnológica de Colombia

- Boyacá - Colombia guadflo@gmail.com

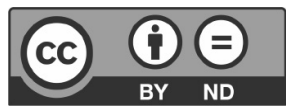


Dentro de los principales hallazgos se destacan: prevalencia de prácticas tradicionales en la enseñanza, poca motivación hacia el aprendizaje desde las competencias de observación, descripción y argumentación, por la ausencia de estrategias desde el Aprendizaje por Indagación.

Palabras clave: Competencias, Ciencias, Educación, Didáctica, Ruralidad. 


\section{Introducción}

Los estándares nacionales buscan el desarrollo de habilidades científicas, que permitan formar personas que no se limiten a acumular conocimientos, sino a aplicarlos a su cotidianidad, haciendo estudiantes competentes y no competitivos MEN (2004, p.5), desafío que los docentes deben adquirir con el fin de formar niños y niñas capaces de producir e indagar, promoviendo una educación ética y crítica que los lleve a comprender su entorno.

En la Institución Educativa Jesús Emilio Jaramillo Monsalve (IEJEJM), a pesar que dentro del currículo y más específicamente en el plan de área de las ciencias naturales, se estipula la formación en competencias de los estudiantes, no se había desarrollado un trabajo que promoviera el desarrollo de competencias científico naturales en los niños y niñas, por lo general se limitaba el trabajo del docente al cumplimiento del plan de área de forma tradicional, dejando de lado los postulados del modelo constructivista que rige los procesos educativos de la institución.

En este sentido y basados en los aportes hechos por Furman (2008, p. 45), quien afirma que las competencias de pensamiento científico no se adquieren de manera espontánea sino que hay que enseñarlas y que el docente y la escuela primaria juegan un rol fundamental en sentar las bases del pensamiento científico, surgió la necesidad de reflexionar alrededor de las prácticas educativas, reconocerlas y comprenderlas desde un análisis profundo.
Con miras a darle un nuevo sentido al trabajo del aula y transformar las prácticas pedagógicas que se orienten a la formación de docentes y estudiantes competentes, reflexivos, críticos, curiosos, creativos, capaces de plantearse preguntas, de interpretar su contexto y de construir conocimiento, se plantea la necesidad de caracterizar los elementos que se incorporan al proceso de enseñanza y aprendizaje alrededor de las competencias científico naturales.

Teniendo en cuenta lo anteriormente expuesto, se pudo concluir que para llevar a cabo el desarrollo del proyecto, fue necesario sentar bases sólidas a partir del diseño y aplicación de instrumentos bien elaborados, que permitieron diagnósticar acertadamente el problema, y a la vez dieron los insumos necesarios para comprender el estado actual de desarrollo de competencias científico naturales desde el aprendizaje por indagación.

Sumado a todo lo anterior, es importante resaltar el papel de la innovación por parte de los docentes en las prácticas pedagógicas y particularmente de las docentes investigadoras, ya que se convirtió en un elemento fundamental que permitió desarrollar y dinamizar un buen proceso de enseñanza, propiciando mejores ambientes de aula, haciendo más efectivo y significativo el aprendizaje y por ende al haber transformaciones en las docentes, estas se vieron reflejadas en los estudiantes (Samacá, 2016).

\section{De las Teorías y Conceptos}

Durante el desarrollo de esta investigación, se trabajó alrededor de
Los estándares nacionales buscan el desarrollo de habilidades científicas, que permitan formar personas que no se limiten a acumular conocimientos, sino a aplicarlos a su cotidianidad, haciendo estudiantes competentes $y$ no competitivos MEN 
tres ejes teóricos que se integraron entre sí, en cada una de las categorías que guiaron el proceso investigativo: Escuela Nueva, Competencias Científicas y Aprendizaje por indagación.

\section{Escuela Nueva}

Propuesta pedagógica, que se referencia en la pedagogía activa, incorpora elementos conceptuales que van de lo activo a lo interactivo, se basa en principios como la construcción social de los conocimientos, contextos que propicien aprendizajes significativos, interacción entre docente, estudiante y conocimiento, atención según ritmos de aprendizaje, evaluación permanente y participativa y desarrollo de competencias (MEN, 2010).

Colbert (2009, p. 45), refiriéndose al papel del docente dentro del modelo Escuela Nueva plantea una serie de críticas en cuanto a la capacitación que se le da a los mismos, menciona que se capacita al docente para aplicar la metodología que debe emplear con sus estudiantes, pero no se le enseña a que él debe cambiar para que el niño aprenda, ya que si solo transmite información, aburre al niño. Enfatiza en las siguientes premisas: memorizando no se aprende, el aprendizaje debe ser participativo, el docente debe facilitar y orientar el proceso, debe plantearle a sus estudiantes preguntas inteligentes que lo conduzcan a analizar, que le permitan descubrir, explorar y aplicar el conocimiento, formar en valores democráticos, fomentar la interacción, el diálogo, el trabajo en equipo. Propicia el aprendizaje basado en el constructivismo.
La escuela nueva está fundamentada en las pedagogías activas, propone líneas de investigación en el campo educativo, incorporando elementos conceptuales de pedagogías contemporáneas que van de lo activo a lo interactivo, señalando horizontes y enfoques para los cuales se establecen metas de formación, que promuevan la construcción social de los conocimientos a través de los contextos, para lograr aprendizajes significativos y la interacción entre estudiantes, docentes y conocimiento dentro y fuera del aula de clases. Atendiendo ritmos de aprendizaje, evaluación de carácter formativa $\mathrm{y}$ participativa, interdisciplinariedad de áreas que contribuya al desarrollo de competencias, de la creatividad y del pensamiento divergente. Para que la pedagogía activa tenga éxito, debe contener dentro de su proceso, maestros con una visión clara de los objetivos hacia donde se dirijan, con la habilidad de conducir a los estudiantes a hacer preguntas, y a pensar críticamente, al igual que generar altas expectativas sobre el rol protagónico de los estudiantes, y su habilidad de tomar responsabilidad en busca de sus propias respuestas dando como resultado un aprendizaje significativo para la vida (MEN, 2010).

\section{Competencias científicas}

Quintanilla (2005, p. 15), afirma que se comienza a hablar de competencias científico naturales, gracias a los cambios que surgen continuamente, en donde las comunicaciones y la tecnología evolucionan, provocando, por consiguiente, que en las escuelas se generen nuevos currículos, nuevos 
modelos pedagógicos y haciendo que la profesión docente requiera ser innovadora, flexible, dispuesta a las transformaciones, aprovechando los contextos culturales. Al respecto del concepto de ciencia, la cataloga como una actividad con finalidades humanas, que requiere de conocimientos coherentes en los cuales el pensamiento científico se identifique con la evolución conceptual; añade que al enseñar ciencia se debe enseñar al estudiante no a resolver problemas científicos sino enseñarle cómo enfrentarse a la resolución de problemas y entender que cada persona aprende ciencia de manera individual y diferente. $\mathrm{Al}$ referirse a las competencias científico naturales, las cataloga como una habilidad para lograr adecuadamente una tarea con ciertas finalidades, conocimientos y motivaciones; que son requisitos para una acción eficaz en el aula en un determinado contexto, a una motivación o un prerrequisito en otro entorno, y al conjunto de saberes técnicos, metodológicos, sociales y participativos, que se actualizan en una situación particular (García-Ramírez \& Martínez-P'érez, 2015).

Carretero (2005, p. 86), menciona que las competencias científico naturales tienen que ver con la indagación, con las curiosidades, con pensar cómo piensan los científicos, ya que el conocimiento no se puede reducir a un conjunto de respuestas predeterminadas.

Al hablar de aprender, sustenta que no es solo la asimilación e incorporación de un conjunto de redes conceptuales, sino cambiar. Por tanto, es necesario y preciso enseñar a cambiar, producir cambios conceptuales y actitudinales, cambios que procedan de la investigación sobre el desarrollo cognitivo de las personas, del estudiante, de la didáctica de las ciencias, aplicados a contextos de enseñanza.

El aprendizaje afecta el desarrollo cognitivo, que es lo que se da en el aprendizaje, es decir lo que se aprende, lo que se reorganiza, por lo tanto, se aprende a experimentar, se aprende mediante una sucesión de cambios conceptuales. En donde todas las ideas previas se conectan de manera directa con el cambio conceptual, Carretero (2005, p. 90). Hay que producir un cambio conceptual en los estudiantes, teniendo en cuenta que ellos son seres humanos con una curiosidad innata, que desde muy pequeños tienen la capacidad de hacer preguntas, es decir son exploradores. Los estudiantes deben ser curiosos, ya que sin preguntas no hay enseñanza de la ciencia, debido a que existe una relación razonable entre las preguntas y las respuestas, aunque a veces las respuestas no tienen el mismo valor que las preguntas y las preguntas no siempre tienen el mismo valor que las respuestas.

Añade además, que es importante centrarse en cómo enseñar las ciencias naturales en la escuela, porque si se está dando un proceso de comprensión, si se consigue que los estudiantes hagan ciencia, que comprendan la ciencia, que representen la realidad y que permitan múltiples lecturas de ésta, se puede decir que se están dando verdaderos procesos educativos y científicos, en los que el estudiante está implicado al demostrar que le interesa, le gusta, 
El aprendizaje por indagación (API), es un modelo didáctico, que toma a las ciencias naturales en sus dos dimensiones como proceso y como producto, que son inseparables y que deben trabajarse a la par siente agrado por estar en la escuela, es decir, se ha conseguido una enseñanza de la ciencia.

Partiendo de los aportes hechos por los anteriores autores, para éste trabajo de investigación se abordaron las siguientes competencias científico naturales, apoyados además, en el trabajo hecho por Furman \& Podestá (2009, p. 72), quienes las clasifican y las definen como se relaciona a continuación.

- Competencia científico natural de observación: Observar va más allá que mirar, se requiere guiar a los estudiantes en los aspectos más importantes del fenómeno, identificando las principales características de los mismos, estableciendo comparaciones. $\mathrm{La}$ observación se orienta hacia un objetivo y por tanto se da en función de ciertos criterios, es un proceso que requiere la utilización de todos los sentidos, ayudados por elementos o instrumentos de medición que potencialicen el uso de los mismos, debido a que el proceso de medición es parte fundamental del proceso de observación, hecho desde diferentes puntos de vista. Un aspecto muy importante a la hora de realizar observaciones es el enseñar a observar y a distinguir entre los datos (el qué) y sus explicaciones (el por qué), ya que se convierte en ficha clave para sentar las bases de las competencias más complejas como lo es la formulación de explicaciones teóricas.

- Competencia científico natural de descripción: La descripción lleva al observador a explicitar los aspectos más significativos del objeto, permite ver sus mentes y comprender como están entendiendo los conceptos. Es parte fundamental de la observación ya que obliga al observador a hacer explícitas las características más significativas del objeto. En los primeros grados de estudio la descripción puede realizarse a través de imágenes o de manera verbal, a medida que va aumentando el grado de escolaridad puede darse de manera escrita.

\section{- Competencia científico natural} de la argumentación: Enseña a los estudiantes a generar consensos sobre su realidad, y a explicar y concluir sobre lo que observan, sobre lo que han investigado. Permite que los estudiantes den razones que sustenten sus afirmaciones, construyan herramientas para tomar decisiones basadas en evidencias y en información relevante. Para que el estudiante sea capaz de argumentar es importante que los docentes construyan en sus clases una cultura en la que promuevan que los estudiantes hagan explícitos sus puntos de vista e intercambien sus ideas con otros, fundamentándolos con evidencias.

\section{Aprendizaje por indagación (API)}

El aprendizaje por indagación (API), es un modelo didáctico, que toma a las ciencias naturales en sus dos dimensiones como proceso y como producto, que son inseparables y que deben trabajarse a la par. Enmarcando los procesos científicos en situaciones de enseñanza en las que el estudiante tenga la oportunidad de desarrollar competencias y conocimiento científico. 
A partir de esta estrategia metodológica el docente lleva un camino predeterminado con sus educandos, en donde se trazan objetivos claros y se construyen conceptos previamente validados por las comunidades científicas, haciendo de las ciencias un proceso activo, dinámico, a partir de la exploración sistemática de fenómenos naturales, el trabajo con problemas y el análisis crítico de experiencias históricas de modo que guarde ciertas analogías con el quehacer científico. Diseña actividades que llevan a los estudiantes a construir conceptos y competencias científico naturales, a partir de planes que se deben adaptar a la dinámica del aula. Para lograr un verdadero proceso de indagación, se requiere que éste proceso sea progresivo y trascendente durante todos los años de estudio, ya que la indagación no se aprende solo con el aprendizaje de conceptos, de métodos, de la generación de hipótesis, debe ser un proceso diseñado a partir de situaciones que desarrollen las competencias científico naturales de manera intencional ya que estas no surgen de manera innata. Es así que el modelo didáctico propone construir el conocimiento a partir de una metodología determinada en donde el conocimiento científico se toma no como un proceso acabado, sino que está en constante cambio, que buscan explicar el funcionamiento del mundo natural, en donde la ciencia se basa en la exploración, las evidencias y la búsqueda de teorías Furman \& Podestá (2009, p. 54).

El API transforma una actividad simple en una oportunidad para enseñar no sólo conceptos, sino competencias científico naturales claves como el diseño experimental, la interpretación de resultados y la argumentación de ideas, respondiendo preguntas sobre el proceso, discutiendo la mejor forma de hacerlo, interpretando sus resultados e intercambiando ideas con otros estudiantes encontrando por ellos mismos la respuesta a un problema.

Tabla 1. Modelo de enseñanza por indagación.

\begin{tabular}{|l|l|}
\hline CONOCIMIENTO CIENTÍFICO & $\begin{array}{l}\text { Es concebido como las construcciones humanas cuyo objetivo } \\
\text { es explicar el funcionamiento de la naturaleza de manera } \\
\text { coherente con la realidad empírica sujeta a cambios. }\end{array}$ \\
\hline METODOLOGÍA DE LA CIENCIA & $\begin{array}{l}\text { Está basada en la exploración sistemática, la búsqueda de } \\
\text { evidencias y la formulación de teorías. }\end{array}$ \\
\hline PAPEL DE LA ESCUELA & $\begin{array}{l}\text { Ofrecer situaciones de enseñanza que promueva en los } \\
\text { estudiantes la curiosidad y hábitos de la pregunta, anticipar } \\
\text { respuestas, generar hipótesis, hacer comparaciones, argumentar, } \\
\text { realizar experiencias que le permitan dar explicaciones } \\
\text { sobre un fenómeno, comparar y confrontar resultados con } \\
\text { sus compañeros. Propiciar ambientes para la producción } \\
\text { de textos orales y escritos, que le permitan dar solución a } \\
\text { problemas cotidianos significativos, generando autonomía en } \\
\text { la construcción del conocimiento. }\end{array}$ \\
\hline ROL DEL DOCENTE & $\begin{array}{l}\text { Diseña actividades que guían al estudiante a construir conceptos } \\
\text { y competencias científico naturales. Se guía por un plan } \\
\text { preestablecido el cual se adapta a la dinámica cambiante del aula. }\end{array}$ \\
\hline ROL DEL ESTUDIANTE & $\begin{array}{l}\text { Participa de manera activa en las actividades realizadas } \\
\text { por el docente, construyendo conceptos y herramientas de } \\
\text { pensamiento científico bajo su tutoría. }\end{array}$ \\
\hline
\end{tabular}

Fuente: Adaptado de la aventura de enseñar ciencias naturales Furman \& Podestá (2009, p. 57). 
El API requiere que el docente diseñe situaciones para enseñar a los estudiantes competencias científicas a partir de los contenidos escolares, para lo cual se requiere del diseño de estrategias que permitan la progresión de los procesos de enseñanza aprendizaje, enseñándolas de manera intencional mediante la planificación de actividades específicas.

El API enseña a observar a los estudiantes para que encuentren los aspectos más relevantes de un fenómeno, guiándolos en la comparación de elementos para que encuentren semejanzas y diferencias y los registren a partir de dibujos o esquemas. Para enseñar a argumentar es necesario diseñar actividades en las que el docente proponga a sus estudiantes el intercambio de puntos de vista diferentes entre estudiantes, soportadas en evidencias que le permitan sustentar sus argumentaciones. Este modelo implica la constante realización de actividades experimentales, que le permitan al estudiante, entrar en contacto con los fenómenos, ya que a través de las experiencias concretas se aporta a la comprensión del mundo, desarrollando el gusto por explorarlo y de interactuar con él a partir de la pregunta. Los experimentos prácticos deben integrarse a un contexto para crear conceptos y herramientas de pensamiento enmarcados en situaciones de enseñanza que les permita sustentarlo. Lo importante al hacer actividades experimentales, es hacer un registro de las preguntas que el estudiante requiere contestar a partir de la experiencia realizada, de las hipótesis planteadas y de los datos recolectados.
Este modelo se basa en la enseñanza integrada de la ciencia como proceso y como producto, esto no implica que el realizar experimentos prácticos en la mayoría de las clases, tampoco implica que una actividad experimental sea la única manera de aprender competencias científico naturales. Por tal razón para planificar una actividad de indagación es necesario identificar los objetivos de enseñanza, diferenciados en conceptos y competencias, priorizándolos dentro de las unidades didácticas a partir de preguntas como, ¿qué es lo más importante del tema? ¿Qué quiero que el estudiante aprenda? Furman \& Podestá (2009, p. 66).

\section{Formulación de preguntas} investigables: "Enseñar a formular preguntas implica que podamos identificar y compartir con los alumnos las preguntas detrás de los temas que estamos enseñando" Furman \& Podestá (2009, p. 79). Para promover en el aula espacios donde la pregunta juegue un rol especial, es necesario que los estudiantes se apropien de ellas, lo cual requiere plantearles situaciones interesantes y con sentido, de fenómenos no familiares que despierten el interés y la curiosidad y que conlleven a generar un gran deseo de entender lo que sucede, dando cabida a la formulación de predicciones e hipótesis.

\section{Del Proyecto Desarrollado}

Este proyecto utilizó la investigación cualitativa en el estudio de los sucesos del aula de clases y la realidad del contexto natural de la escuela en donde 
se desarrollaron los procesos educativos tal y como ocurrían, se interpretaron los datos recogidos a través de los diferentes instrumentos, como la encuesta y el cuestionario, los cuales por su parte, tuvieron por objeto en el caso de la encuesta, recoger información acerca del proceso de integración de las competencias científico naturales en la enseñanza de las ciencias naturales, y en el caso del cuestionario conocer la forma en cómo se enseñaban y se aprendian las ciencias naturales en la escuela permitiendo comprender las situaciones que vivía cada uno de los sujetos implicados en la investigación, las estrategias utilizadas por los docentes y el grado de desarrollo de las competencias científico naturales (observación, descripción, argumentación) de los estudiantes de cuarto y quinto grado, al igual que el papel de la indagación en la enseñanza de las ciencias naturales. Las preguntas planteadas se formularon en función de las categorías y subcategorías de análisis, propuestas para el desarrollo de la investigación.

Los instrumentos fueron construidos a partir de acuerdos o consensos fundamentados en el diálogo entre varios actores del proceso investigativo, haciendo posible la interacción y cooperación estrecha entre investigador y agentes sociales, quienes a partir de esta, construyeron perspectivas de comprensión más completas y de transformación más factibles. En este caso, la indagación fue guiada, se estructuró a partir de los hallazgos que se fueron encontrando durante el desarrollo de la misma, las conclusiones se construyeron a partir de los hallazgos y de los resultados arrojados por los instrumentos, los cuales teniendo en cuenta a Casilimas (2002, p.35) en el transcurso de la investigación se concretaron a partir de procesos de observación, reflexión, diálogo, construcción de sentido y sistematización.

En este trabajo se utilizó la investigación acción (IA) como método investigativo, ya que permitió a las docentes estudiar los contextos educativos, para identificar la problemática más sentida por la comunidad, interpretar lo que ocurría dentro y fuera del aula de clase, describir los procesos que se daban en el entorno educativo y proponer soluciones, a través de la implementación de proyectos fruto de la investigación, que ayudarán a la transformación de las prácticas pedagógicas, gracias a la interacción continua entre los diferentes actores implicados.

La IA abordó los problemas prácticos de la vida cotidiana experimentados por las docentes, su propósito consistió en hacer un diagnóstico de la problemática adoptando una postura investigativa relacionándola con el contexto. Interpretando lo que ocurría desde el punto de vista de quienes intervinieron en la situación problema, es decir, docentes y estudiantes Elliott (2005, p.74).

Teniendo en cuenta la población con la cual se trabajó se consideró la aplicación de dos instrumentos, la encuesta y el cuestionario, los cuales se elaboraron atendiendo a los objetivos del proceso investigativo, con el fin de
En este trabajo se utilizó la investigación acción (IA) como método investigativo, ya que permitió a las docentes estudiar los contextos educativos, para identificar la problemática más sentida por la comunidad, interpretar lo que ocurría dentro y fuera del aula de clase 
recolectar información puntual sobre el contexto, las prácticas pedagógicas y a la vez permitirán plantear las estrategias a trabajar durante el proceso investigativo.

Para ésta investigación se definieron tres categorías en función del objeto de investigación que son Escuela Nueva, Competencias Científico Naturales y Aprendizaje por Indagación.

\section{Del Reconocimiento de Contexto}

Con el propósito de caracterizar los elementos que se incorporaban al proceso de enseñanza aprendizaje alrededor de las competencias científicas se desarrollaron cinco actividades:

\section{ACTIVIDAD 1. Del Contexto Escolar}

La IEJEJM, es una institución rural de carácter público, ubicada al sur del municipio de Chita a ocho Kilómetros del casco urbano, tiene varias vías de acceso entre ellas tres carreteables en regular estado y varios caminos de herradura. En su entorno predomina el clima frío y paramuno, se destaca el minifundio, la economía se sustenta en la agricultura con productos tradicionales con una escala de producción poco tecnificada y la ganadería extensiva en menor proporción, sus suelos son fértiles, con abundante riqueza hídrica y cuenta con un distrito de riego. La comunidad educativa la conforman padres de familia y estudiantes campesinos, sus principales actividades económicas dependen del trabajo agrícola y pecuario, conservan sus tradiciones (religión, fiestas, política, comidas, creencias, costumbres, folclor) y en general a la cultura campesina. La mayoría de ellos son personas de escasos recursos económicos pertenecientes al estrato 1.

La comunidad educativa está conformada por un directivo, once docentes, un auxiliar administrativo (secretaria), 95 padres de familia y 204

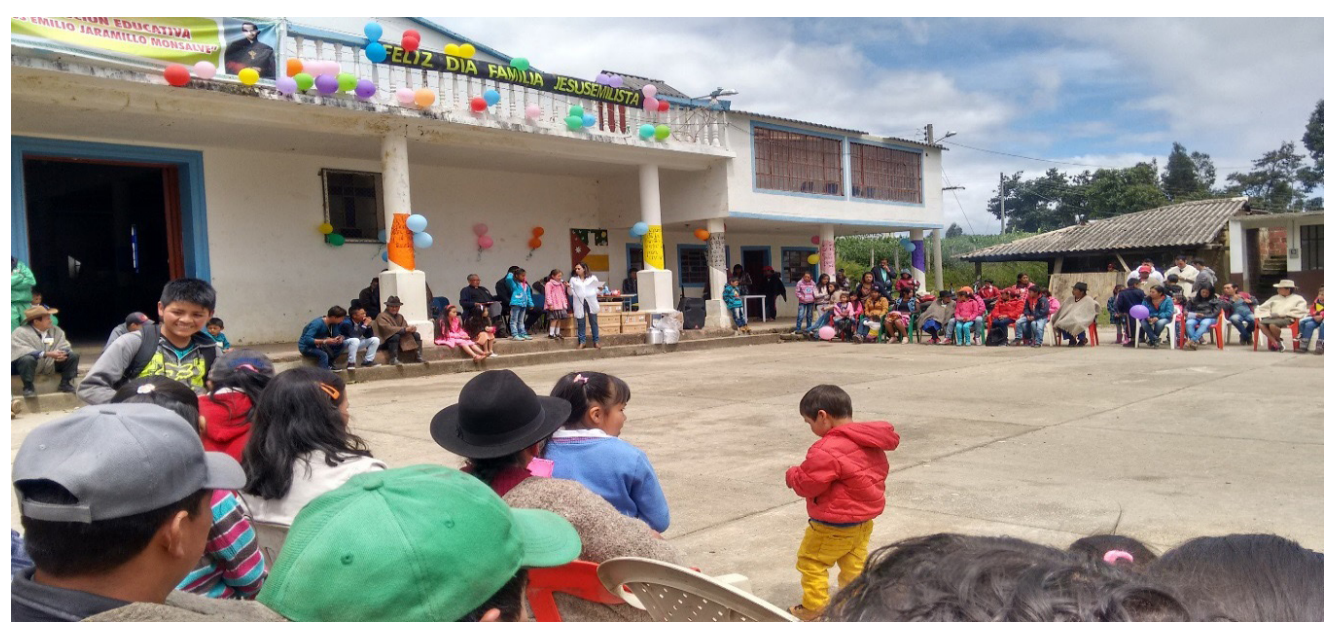

Fuente: Autoras 2017 
estudiantes. En la IEJEJM se ofrecen los niveles educativos determinados por la ley 115 de 1994, entre los cuales están: Preescolar, básica primaria, básica secundaria, media vocacional, educación para adultos con el programa S.O.S y además cuenta con un convenio establecido con el SENA en producción pecuaria.

\section{ACTIVIDAD 2. De los Participantes}

La población con la cual se desarrollará el proyecto, son 204 niños y jóvenes de la IEJEJM, que oscilan entre las edades de 5 a 19 años, provenientes de diferentes veredas circunvecinas como Chipa Centro, Chipa Betel, La Estancia, Cuco, Vichacuca, Chipa Alto, $\mathrm{y}$ algunos estudiantes que van desde el casco urbano del municipio de Chita. En el nivel de preescolar cuenta con un número de 12 estudiantes, en el nivel de primaria 69 estudiantes y en el nivel de secundaria cuenta con un número de 123 estudiantes. Tiene un total de 11 docentes distribuidos de la siguiente manera: 3 en primaria, 8 en bachillerato y un directivo. En su parte administrativa se cuenta con una auxiliar.

Se seleccionó por conveniencia de las investigadoras los 27 estudiantes, de los grados cuarto (16) y quinto (11) de primaria, dado que son las directoras de grado de estos cursos y orientan el proceso de formación de dichos estudiantes, cuyas edades oscilan entre los 9 y los 13 años, provenientes de familias nucleares en menor proporción, monoparentales y extensas en su mayoría, de escasos recursos económicos y cuyos padres poseen un nivel de estudio que no supera el nivel de educación de básica primaria.

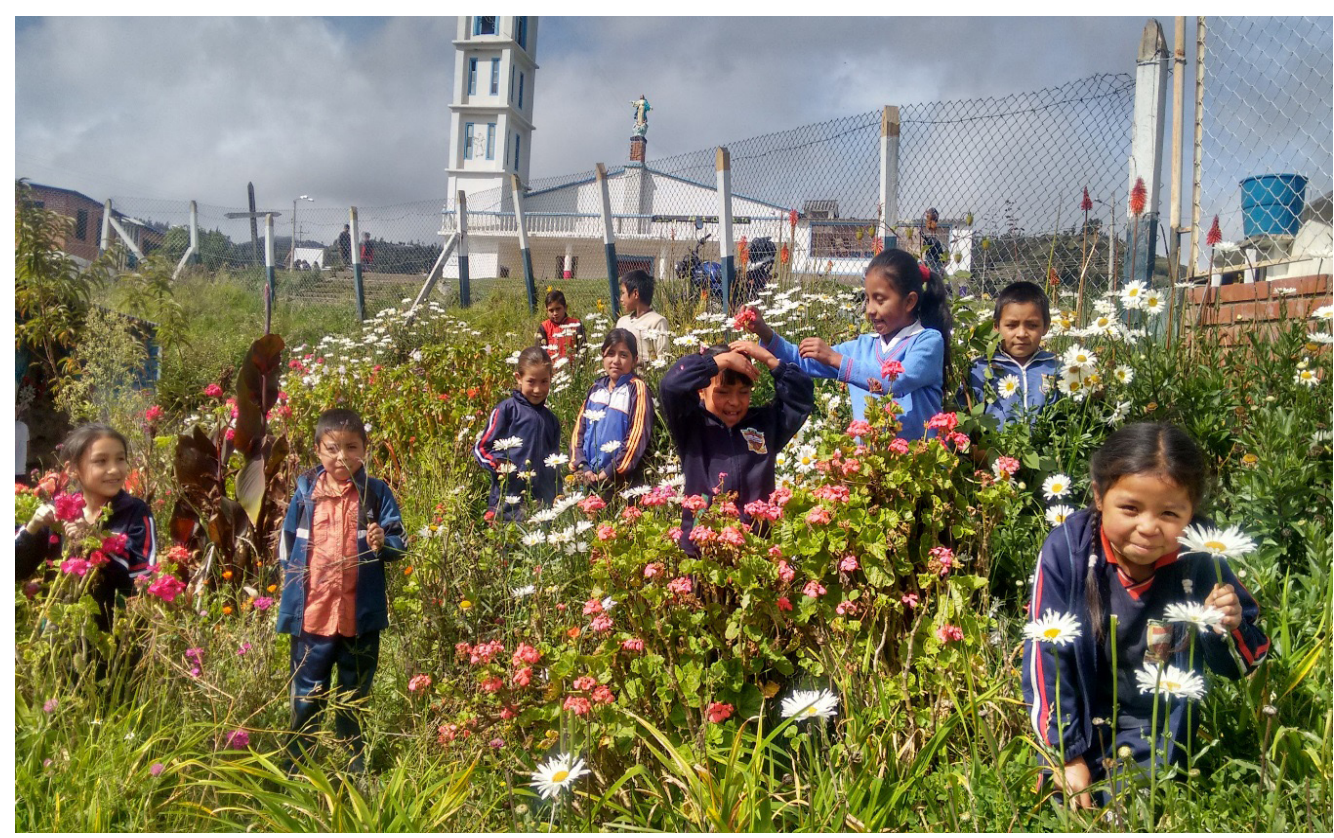

Fuente: Autoras 2017 


\section{ACTIVIDAD 3. De la Construcción de categorías y subcategorías.}

Para ésta investigación se definieron tres categorías, escuela nueva, competencias científicas y aprendizaje por indagación.

Tabla 2. Descripción de la intensión de las categorías con sus subcategorías.

\begin{tabular}{|c|c|c|c|}
\hline Categoría & Definición & Subcategoría & Definición \\
\hline \multirow[b]{2}{*}{$\begin{array}{c}\text { Modelo } \\
\text { Pedagógico } \\
\text { Escuela Nueva }\end{array}$} & \multirow{2}{*}{$\begin{array}{l}\text { Modelo pedagógico que } \\
\text { privilegia la construcción social } \\
\text { del conocimiento, desde la } \\
\text { interacción docente-estudiante. } \\
\text { Con ésta categoría se evidencia } \\
\text { el grado de incorporación de } \\
\text { las competencias en el contexto } \\
\text { rural donde se ubica la escuela } \\
(\mathrm{MEN}, 2010) \text {. }\end{array}$} & Currículo & $\begin{array}{l}\text { Currículo flexible, el cual } \\
\text { contempla estrategias } \\
\text { específicas que permiten dar } \\
\text { respuesta a las necesidades } \\
\text { de los niños (MEN, 2009). }\end{array}$ \\
\hline & & Contexto rural & $\begin{array}{l}\text { Espacio a través del cual } \\
\text { se implementan opciones } \\
\text { educativas pertinentes que } \\
\text { promueven la articulación } \\
\text { de la educación al desarrollo } \\
\text { productivo y social (MEN, } \\
2001 \text { ). }\end{array}$ \\
\hline \multirow{3}{*}{$\begin{array}{l}\text { Competencias } \\
\text { científico } \\
\text { naturales }\end{array}$} & \multirow{3}{*}{$\begin{array}{l}\text { Las competencias científico } \\
\text { naturales enmarcan los modos } \\
\text { de conocer las ciencias naturales } \\
\text { a través de la observación, la } \\
\text { descripción y la argumentación, } \\
\text { la formulación de preguntas, } \\
\text { el diseño de actividades } \\
\text { experimentales que permiten } \\
\text { formular hipótesis acerca de } \\
\text { fenómenos (Furman \& Podesta, } \\
\text { 2009). Esta categoría permite } \\
\text { conocer el nivel de desarrollo } \\
\text { de las competencias científico } \\
\text { naturales en los estudiantes. }\end{array}$} & $\begin{array}{l}\text { Competencia } \\
\text { Observación }\end{array}$ & $\begin{array}{l}\text { La observación le permite } \\
\text { al estudiante destacar los } \\
\text { aspectos más importantes } \\
\text { de los fenómenos, hacer } \\
\text { comparaciones, establecer } \\
\text { generalizaciones, a partir } \\
\text { de preguntas investigables } \\
\text { propuestas por el docente y } \\
\text { los mismos estudiantes. }\end{array}$ \\
\hline & & $\begin{array}{l}\text { Competencia } \\
\text { Descripción }\end{array}$ & $\begin{array}{l}\text { Competencia que permite } \\
\text { a los estudiantes hacer } \\
\text { descripciones verbales o } \\
\text { escritas de los fenómenos, a } \\
\text { través del uso de imágenes y } \\
\text { textos. }\end{array}$ \\
\hline & & $\begin{array}{l}\text { Competencia } \\
\text { Argumentación }\end{array}$ & $\begin{array}{l}\text { Esta subcategoría permite } \\
\text { a los estudiantes dar } \\
\text { razones que sustenten sus } \\
\text { afirmaciones y les permitan } \\
\text { tomar decisiones basadas en } \\
\text { evidencias y en información } \\
\text { relevante. }\end{array}$ \\
\hline
\end{tabular}




\begin{tabular}{|c|c|c|c|}
\hline \multirow{3}{*}{$\begin{array}{l}\text { Aprendizaje por } \\
\text { indagación }\end{array}$} & \multirow{3}{*}{$\begin{array}{l}\text { El API es una estrategia } \\
\text { didáctica que toma a las } \\
\text { ciencias naturales en sus dos } \\
\text { dimensiones como proceso y } \\
\text { como producto, y permite el } \\
\text { desarrollo de competencias y } \\
\text { conocimiento científico a partir } \\
\text { de la exploración de fenómenos } \\
\text { (Furman \& Podesta, 2009). } \\
\text { Esta categoría fundamenta } \\
\text { la estrategia didáctica a } \\
\text { ser aplicada, ya que busca } \\
\text { promover competencias } \\
\text { científico naturales en los } \\
\text { estudiantes de los grados cuarto } \\
\text { y quinto de la IEJEJM. }\end{array}$} & $\begin{array}{l}\text { Ambiente de } \\
\text { aula }\end{array}$ & $\begin{array}{l}\text { Esta subcategoría permite } \\
\text { reconocer el valor } \\
\text { que se le atribuye a la } \\
\text { generación de ambientes } \\
\text { de aula agradables para el } \\
\text { desarrollo del proceso de } \\
\text { enseñanza aprendizaje de } \\
\text { las competencias científico } \\
\text { naturales. }\end{array}$ \\
\hline & & Didácticas & $\begin{array}{l}\text { A partir de esta subcategoría } \\
\text { se diseñan talleres, } \\
\text { actividades experimentales, } \\
\text { unidades didácticas, que } \\
\text { permiten desarrollar las } \\
\text { competencias científico } \\
\text { naturales de los estudiantes. }\end{array}$ \\
\hline & & $\begin{array}{c}\text { Problematiza- } \\
\text { ción }\end{array}$ & $\begin{array}{l}\text { Esta subcategoría permite } \\
\text { formular preguntas } \\
\text { investigables, que plantean } \\
\text { situaciones que resultan } \\
\text { interesantes y significativas } \\
\text { para los estudiantes. }\end{array}$ \\
\hline
\end{tabular}

Fuente: Autoras 2017

\section{ACTIVIDAD 4. Del Diseño y aplicación de instrumentos:}

Se diseñaron y validaron los instrumentos utilizados en la investigación. Se aplicaron encuestas y cuestionarios, tanto a docentes como a estudiantes, con el fin de obtener información relevante que permitió plantear las estrategias adecuadas para el desarrollo de la investigación.

Tabla 3. Descripción de la intensión de los instrumentos utilizados en la investigación.

\begin{tabular}{|c|c|c|}
\hline INSTRUMENTO & CONCEPTO & INTENCIÓN \\
\hline ENCUESTA & $\begin{array}{l}\text { Este instrumento se diseñó con el } \\
\text { propósito de recoger datos a partir de } \\
\text { la formulación de preguntas abiertas } \\
\text { y cerradas que permitieron conocer } \\
\text { aspectos relacionados con las estrategias } \\
\text { de enseñanza y el ambiente de aula de } \\
\text { las clases de ciencias naturales (Casas, J., } \\
\text { Repullo, J., \& Donado J., 2003). }\end{array}$ & $\begin{array}{l}\text { La encuesta permitió conocer las } \\
\text { estrategias didácticas utilizadas para la } \\
\text { enseñanza de las ciencias naturales, al } \\
\text { igual que el ambiente escolar en el que } \\
\text { se llevaba a cabo el aprendizaje. }\end{array}$ \\
\hline CUESTIONARIO & $\begin{array}{l}\text { Las preguntas del cuestionario estuvieron } \\
\text { dadas a partir del planteamiento de } \\
\text { situaciones, que ayudaron a identificar } \\
\text { las competencias científico naturales que } \\
\text { los estudiantes desarrollaban (Sampieri, } \\
\text { R., Collado, C. y Lucio, P., 2003). }\end{array}$ & $\begin{array}{l}\text { El cuestionario permitió conocer el } \\
\text { nivel de desarrollo de las competencias } \\
\text { científico naturales de observación, } \\
\text { descripción y argumentación. }\end{array}$ \\
\hline
\end{tabular}

Fuente: Autoras 2017 


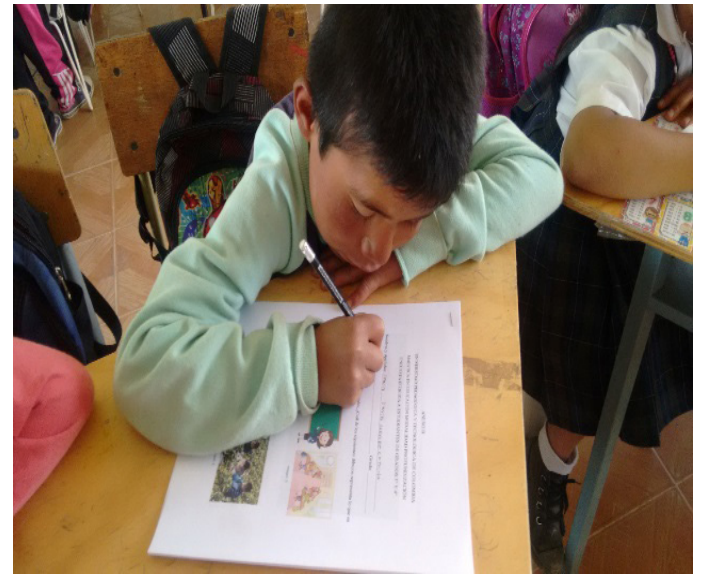

Fuente: Autoras 2017 Fuente: Autoras 2017

ACTIVIDAD 5. Análisis a partir de la triangulación

Se realizó un análisis y tabulación de los datos recogidos a partir de la aplicación de los instrumentos con base en las categorías previstas.

\section{Categoría: Escuela Nueva}

Se puede interpretar a partir de los resultados que las clases de ciencias naturales son desarrolladas por los docentes utilizando metodologías tradicionalistas, en donde el estudiante juega un papel pasivo y receptivo, $y$ el aula es el lugar privilegiado para el desarrollo de éstas. A pesar de que se trabaja el modelo escuela nueva, el cual nace del constructivismo, el trabajo desarrollado no da muestras de los principios que rigen la misma, $\mathrm{y}$ aunque en los planes de áreas se habla de desarrollo de competencias son muy escasas las actividades encaminadas al fortalecimiento de las mismas, esto se evidencia en las respuestas dadas por los docentes, ya que la enseñanza de las ciencias naturales en la institución

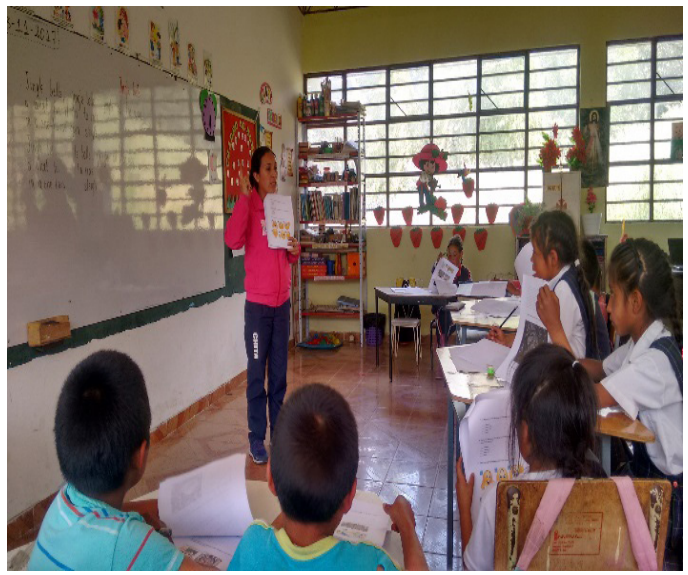

está supeditada a factores relacionados con el material didáctico y la temática del área, dejando de lado el contexto como elemento fundamental para la construcción del conocimiento. Al respecto el MEN, 2010 plantea la importancia de incorporar elementos conceptuales basados en la construcción social de conocimientos, en la promoción de contextos que propicien aprendizajes significativos atendiendo a ritmos de aprendizaje $y$ que sea un proceso permanente en donde se privilegie el desarrollo de competencias.

Aunque los niños provienen de hogares campesinos, el entorno del que proviene el estudiante no está siendo aprovechado al máximo, debido a que se le da un sentido utilitarista y no se evidencia como un medio para el proceso formativo y de apropiación de las ciencias naturales, dejando de lado la importancia del papel que juega el contexto dentro del proceso educativo. $\mathrm{Al}$ respecto y de acuerdo a lo planteado por el MEN, 2010, el contexto del estudiante debe promover aprendizajes 
significativos, convirtiéndose en un factor importante que se debe tener en cuenta en el desarrollo de competencias. Por su lado Flórez, 2000, privilegia dentro de los principios que rigen la escuela nueva la experiencia natural y dentro de la misma la espontaneidad del niño, la cual debe atender a las necesidades, intereses del mismo con el fin de enriquecer los procesos y hacerlos más significativos Atendiendo a lo anterior y teniendo en cuenta los datos obtenidos se puede evidenciar que los docentes conocen el contexto en el cual se encuentra ubicada la institución y refieren aprovecharlo en el desarrollo de las temáticas abordadas en las clases de ciencias naturales, resaltan los valores culturales de la región y dicen aprovecharlo en el proceso de enseñanza que llevan a cabo.

\section{Competencias científico naturales}

De acuerdo a la información obtenida los estudiantes en su gran mayoría realizan procesos deficientes de observación, ya que sólo se detienen en aspectos superficiales de los fenómenos o las cosas, no realizan procesos detallados de los mismos, debido a la falta de claridad sobre lo que se pretende estudiar de estos, en este sentido cabe mencionar los aportes de Furman \& Podestá, 2009, quienes manifiestan que el desarrollo de competencias científico naturales en los estudiantes les han de permitir comprender los fenómenos que ocurren a su alrededor.

En lo que respecta al docente y según lo analizado hay confusión en el concepto de competencias científico naturales, la mayoría de docentes plantean tan solo actividades que pueden ayudar al fortalecimiento de estas pero presentan dificultad al definir el significado de las mismas. No se evidencia que se desarrolle un proceso secuencial que propenda por el desarrollo de competencias, en este sentido al hablar de competencias científico naturales Carretero (2005) resalta la importancia que tiene el desarrollar la indagación y la curiosidad en los primeros años de estudio.

En cuanto al proceso de descripción se puede interpretar que el trabajo descriptivo realizado por los estudiantes va aumentando de acuerdo al grado de escolaridad y la edad de los mismos, convirtiéndose en un trabajo más riguroso y preciso, en el cual se tienen en cuenta características específicas del objeto.

Partiendo del análisis de las respuestas dadas por los docentes, éstos dan razón de cómo promueven la competencia de observación, más no de la competencia descripción con sus estudiantes, en las clases impartidas de ciencias naturales, aunque en la mayoría de los casos parece que recaen en el activismo. En virtud de ello, es necesario guiar el proceso de descripción en los estudiantes llevando a que éstos establezcan características que les permitan explicitar los aspectos más significativos del fenómeno, teniendo en cuenta que la descripción y la observación son dos procesos que se encuentran intrínsecamente relacionados (Furman \& Podestá, 2009). 
Con relación a la argumentación hay algunos estudiantes que plantean hipótesis acertadas y dan cuenta de fenómenos, basados en experiencias personales, en saberes obtenidos en las clases y de personas allegadas como los abuelos, padres, etc. El grado de argumentación es superficial en la mayoría de estudiantes debido a que son muy pocas las razones con las cuales defienden sus hipótesis, es limitado y está sujeto a ideas sueltas.

No se evidencia un proceso de análisis y de argumentación claro que permita desarrollar en los estudiantes esta competencia científico natural, ya que el trabajo se basa en la toma de opiniones y respuestas a problemas por parte de los estudiantes de forma superficial y poco analítica. En contraste con los datos obtenidos es necesario tener en cuenta la importancia que tiene la competencia de argumentación ya que ayuda al estudiante a explicar y concluir sobre lo que observa, sobre su realidad, permitiendo que dé razones que le ayuden a tomar decisiones basado en evidencias y en información relevante que adquiere después de haber pasado por un proceso de observación y descripción y desde luego para que se dé un proceso de argumentación en el aula es necesario que el docente promueva espacios en donde el estudiante tenga la oportunidad de intercambiar ideas fundamentadas con evidencias y las confronte con sus pares (Furman, 2009).

\section{Aprendizaje Por Indagación}

Con relación al análisis hecho a los resultados, las estrategias utilizadas por las docentes están limitando el trabajo de los estudiantes, debido a que el rol del estudiante tiende a ser pasivoreceptivo, y las clases tienen tendencia a utilizar modelos tradicionalistas. Por otro lado se interpreta que no se proponen alternativas didácticas que dinamicen los procesos desarrollados dentro y fuera del aula de clases, ya que los docentes se limitan a mencionar algunas acciones que desarrollan en sus clases, pero no hay una definición clara de la estrategia didáctica empleada por los mismos para el área en específico, es decir no hay claridad en cuanto a la metodología empleada por algunos docentes en el área de ciencias naturales.

En este sentido cabe mencionar el papel que desempeña el Aprendizaje por Indagación ya que es una estrategia que transforma una actividad simple en una oportunidad para enseñar no sólo conceptos sino competencias científicas a través de actividades como el diseño experimental, la interpretación de resultados y la argumentación de ideas (Furman \& Podestá, 2009), que ayuda a dinamizar y a hacer más significativos los procesos tanto de enseñanza como de aprendizaje.

Otro de los hallazgos encontrados a partir de las respuestas dadas tanto de estudiantes como de docentes es que no se privilegia la pregunta dentro de las clases de ciencias naturales, en el caso del docente debido a que los temas son desarrollados con el ánimo de promover temáticas de forma rápida y la pregunta en algunos casos es utilizada como método de represión hacia el estudiante, sobre todo cuando 
se presenta indisciplina, $y$ en otras ocasiones se formulan preguntas fácticas que sólo buscan conceptos, no buscan generar inquietud, ni enseñar a pensar al estudiante, y por ende podría decirse que no desarrollan competencias, y en el caso del estudiante por miedo a equivocarse, por timidez, o por evitar el bulling, ya que puede ser tomada como sinónimo de ignorancia. Se interpreta además que los docentes no dan una respuesta clara a la subcategoría de problematización, puesto que algunos hacen referencia a material didáctico, otros a las limitaciones que tienen en sus clases, tan solo un docente toma lo cotidiano como punto de partida para el desarrollo de las temáticas.

En cuanto a éste hallazgo cabe citar lo mencionado por Furman \& Podesta (2009), en lo que se refiere a los procesos de indagación, mencionan que se requiere que sean progresivos y se den durante toda la etapa escolar, además deben partir de situaciones que desarrollen competencias científico naturales ya que éstas no surgen de manera innata, y en donde la pregunta se convierta en un elemento fundamental dado que a través de ésta se genera conocimiento y se producen aprendizajes significativos cuando se hacen de manera inteligente. En palabras de Carretero, 2005 sin preguntas no hay enseñanza de la ciencia.

Otro aspecto que se analizó es el relacionado con el ambiente de aula, los estudiantes demuestran agrado y gusto por el área de ciencias naturales, gracias tal vez a la interacción que tienen a diario con los elementos naturales que los circundan, a pesar de que las docentes desarrollan las temáticas en un mismo espacio y manejan una metodología tradicionalista privilegiando la transcripción textual de los contenidos. Además, según los resultados obtenidos se interpreta que los docentes refieren que el trabajo de campo y el trabajo experimental son esenciales para el desarrollo de las clases de ciencias naturales, dejando de lado procesos fundamentales como la observación, la descripción y la argumentación, y que los recursos didácticos son su prioridad, pero no se tienen en cuenta los demás elementos que hacen parte del ambiente de aprendizaje, como la implementación de nuevas estrategias, metodologías y la importancia que tiene hacer una resignificación de las prácticas pedagógicas, tan solo un docente toma en cuenta aspectos relevantes del ambiente de aula y lo relaciona con el contexto.

En virtud de lo anterior se ve la necesidad de propiciar espacios y situaciones que promuevan en los estudiantes la curiosidad, el hábito de la pregunta, la argumentación, la experimentación, la observación, con el objeto de que le permitan dar solución a problemas cotidianos significativos y se genere autonomía en la construcción del conocimiento ((Furman \& podestá, 2009).

\section{A manera de cierre}

A partir de la construcción, aplicación y análisis de los instrumentos se logró 
conocer de forma precisa y veraz el nivel de desarrollo en el que se encuentran las competencias científico naturales en los estudiantes y el nivel de conocimiento y manejo de las mismas por parte de los docentes de la Institución Educativa JEJM.

Se pone en evidencia que las estrategias que emplean los docentes para la enseñanza de las ciencias naturales en la institución se encuentran enmarcadas en metodologías tradicionalistas, y a pesar de que se realizan actividades que podrían ayudar a desarrollar competencias científico naturales en los estudiantes no hay claridad en el proceso, lo que devela un campo fundamental para los procesos investigativos desde la didáctica de las ciencias naturales en la apropiación de estrategias como las que plantea el Aprendizaje por Indagación.

En función de esto, es importante darle valor a la formulación de preguntas investigables que propone el API, ya que resulta indispensable para el proceso de aprendizaje de los estudiantes y la apropiación de las competencias científicas, motivando adicionalmente en los niños y jóvenes la curiosidad, la creatividad y la innovación, que sumado a esto con el contexto rural, brindará nuevos derroteros para seguir transformando las prácticas educativas alrededor de la enseñanza y el aprendizaje de las ciencias naturales.

\section{Referencias Bibliográficas}

Carretero, M. (2005). Foro Educativo de Competencias Cientificas. Conocimiento, aprendizaje y desarrollo de competencias cientificas en la escuela (págs. 85-101). Bogotá, Colombia: Ministerio de educación Nacional.

Casas, J., Repullo, J., \& Donado J. (2003). La encuesta como técnica de investigación. Elaboración de cuestionarios y tratamiento estadístico de los datos. Madrid, España: ELSEVIER.

Casilimas, C. A. (2002). Investigación Cualitativa. Bogotá, Colombia: ARFO editores e impresores.

Colbert, V., Bery, L., \& Mogollón, O. (2009). Escuela Nueva - Escuela Activa. Manual para el docente. Bogotá, Colombia: Fundación Volvamos a la Gente.

Elliott, J. (2005). El Cambio Educativo desde la Investigación-Acción. Madrid, España: Ediciones Morata.

Furman, M. (2008). IV Foro Latinoamericano de Educación. Ciencias naturales en la escuela primaria: Colocando las piedras fundamentales del pensamiento científico, Bogotá, Colombia: Fundación Santillana 
Furman, M., \& Podestá, M. E. (2009). La Aventura de Enseñar Ciencias Naturales. Argentina: AIQUE Educación.

García-Ramírez, N., \& Martínez-Pérez, L. (2015). Incidencia del abordaje de una cuestión socio-científica en la alfabetización científica y tecnológica de jóvenes y adultos. . Praxis \& Saber, 6(11), 87 - 114. https://doi.org/10.19053/22160159.3576

MEN. (1998). Serie de Lineamientos Curriculares. Santafé de Bogotá, Colombia: Ministerio de Educación Nacional.

MEN. (2001). Revista al Tablero $\mathrm{N}^{\circ}$ 2. El periódico de un país que educa y se educa. Bogotá, Colombia: Ministerio de Educación Nacional.

MEN. (2004). Estándares Básicos de Competencias en Ciencias Naturales y Ciencias Sociales. Primera edición. Bogotá, Colombia: Ministerio de Educación Nacional.

MEN. (2009). Escuela Nueva - Escuela Activa. Manual para el docente. Bogotá, Colombia: Fundación Volvamos a la Gente.

MEN. (2010). Manual de Implementación Escuela Nueva Generalidades y Orientaciones Pedagógicas para Transición y Primero. Tomo 1. Bogotá, Colombia: Ministerio de Educación Nacional.

IEJEJM. (2014). Proyecto Educativo Institucional. Chita, Colombia: Institución Educativa Jesús Emilio Jaramillo Monsalve

Quintanilla, M. (2005). Foro Educativo de Competencias Científicas. Qué y Cúales son las Competencias Cientificas (págs. 13-30). Bogotá, Colombia: Ministerio de Educación Nacional.

Samacá, I. (2016). El espíritu científico en la primera infancia. Praxis \& Saber, 7(13), 89 - 106. https://doi.org/10.19053/22160159.4167

Sampieri, R., Collado, C. \& Lucio, P., (2003). Metodología de la Investigación. McGraw-Hill Interamericana. México, D.F. 